\title{
MODELLING TIME-DEPENDENT DIFFUSION IN AM STAR ENVELOPES
}

\author{
G. ALECIAN \\ DAEC, URA173-CNRS,Observatoire de Paris, Section de Meudon, \\ F-92195 MEUDON CEDEX, FRANCE
}

\begin{abstract}
We study the diffusion process occurring just below the superficial convection zone of Am stars, improving the methods used sofar. We are now able to compute, in a more realistic way, the evolution of the concentrations during the stay of the stars on the main sequence for a large number of elements. This allows to better constrain the different properties (mass loss, depth of the superficial convection zone, transition between convection and radiative zone) entering the modelling of Am stars in the framework of a diffusion-dominant description.
\end{abstract}

\section{NUMERICAL COMPUTATION OF THE TIME-DEPENDENT DIFEUSION}

The diffusion process, in addition to the global mass flow (due to the stellar massloss), stratifies elements inside the stars. We have solved the continuity equation for the concentrations below the superficial convection zone using an improved version of the code of Alecian $(1986,1990)$.

The radiative accelerations are determined through the detailed computation of the number of photons absorbed by the ions of interest. This needs to often deal with a great amount of atomic data (here the data of Kurucz and Peytremann, 1975). To compute these accelerations we have used the method proposed by Alecian \& Artru (1990).

\section{STELLAR ENVELOPE MODELS}

We have computed the envelope models for two effective temperatures $(7400 \mathrm{~K}$ and $8000 \mathrm{~K}$ ) with $\log \mathrm{g}$ around 4.0 , which correspond to the Am stars' characteristics. We have also decided to impose a low concentration of helium $(\mathrm{Y}=0.08)$ since $\mathrm{He}$ is supposed to be deficient in these stars (deficiency due to gravitational settling).

There are two important parameters concerning the diffusion process: the rate of mass loss and, the thickness of the superficial convection zone. The rate of mass loss may be considered as a free parameter since there is not any observational data about it for Am stars. On the contrary, the position of the superficial convection zone is not a free parameter. However, on the one hand there is some uncertainty in its determination and, on the other hand, the bottom of the convection zone may be followed by a thin mixing zone (overshooting, internal waves,...). 
We have taken into account this uncertainty and we have quantified it through an exponential law with respect to the depth.

TABLE I The different cases (models) which have been considered

\begin{tabular}{ccccc}
\hline $\mathrm{N}^{\circ}$ & $\mathrm{T}_{\text {eff }}$ & $\log \mathrm{g}$ & $\begin{array}{c}\text { Extension of the } \\
\text { superficial CZ }\end{array}$ & $\begin{array}{c}\text { mass-loss } \\
\text { (In solar mass per year) }\end{array}$ \\
\hline 1 & $8000 \mathrm{~K}$ & 3.94 & no & $1.10^{-14}$ \\
2 & & & no & $3.10^{-14}$ \\
3 & & & yes & $1.10^{-14}$ \\
\hline 4 & $7400 \mathrm{~K}$ & 4.05 & no & $1.10^{-14}$ \\
\hline
\end{tabular}

\section{DISCUSSION}

We have considered three elements: $\mathrm{Al}, \mathrm{Ca}, \mathrm{Sc}$ which present abnormal concentrations in Am stars. The time-dependent diffusion of these 3 elements has been computed (according to the different cases shown in Table I).

Our main conclusions are the following:

(i) - Elements go through successive phases of under/overabundances during the life of the star on the main sequence. We stress on the "long" computation for Ca (Fig. 1) which shows that one second small phase of overabundance is followed by a third phase of underabundance after less than one billion years of diffusion.

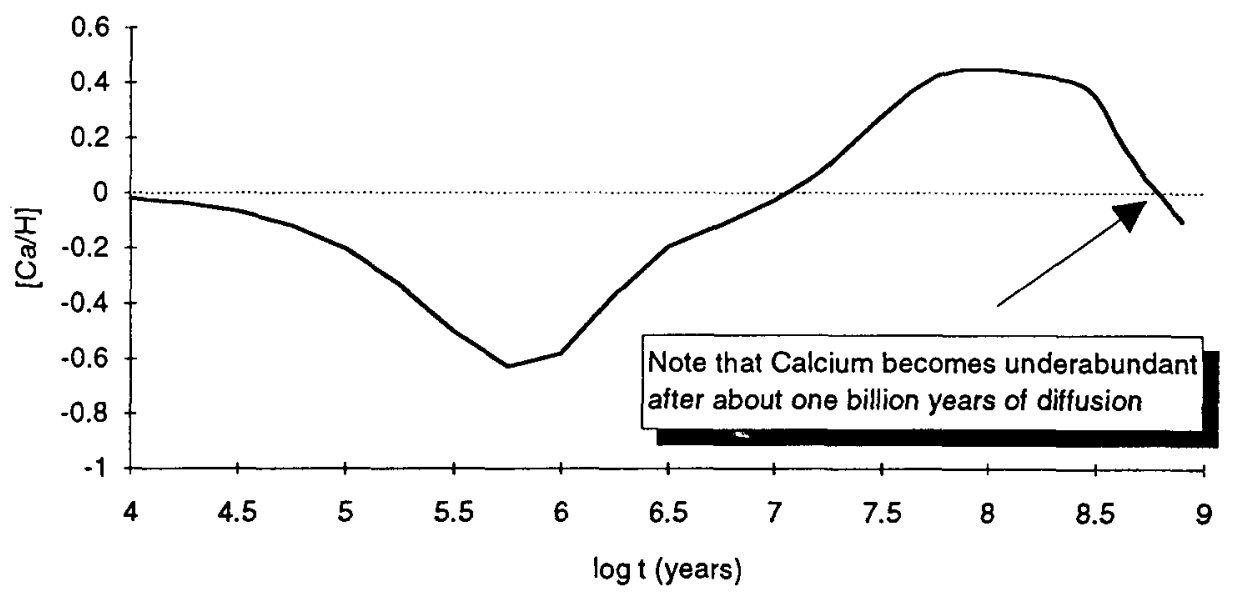

Fig. 1. Calcium aboundance during $10^{9}$ years of diffusion (model 4).

(ii) - Some elements are more sensitive than others on the mass loss rate, depth of the convection zone or effective temperature (Fig. 2). However, in most cases the mass loss rate seems to be the most crucial one. Our computations suggest that the critical value for this rate may be around $10^{-14}$ solar mass per year for Am stars. 


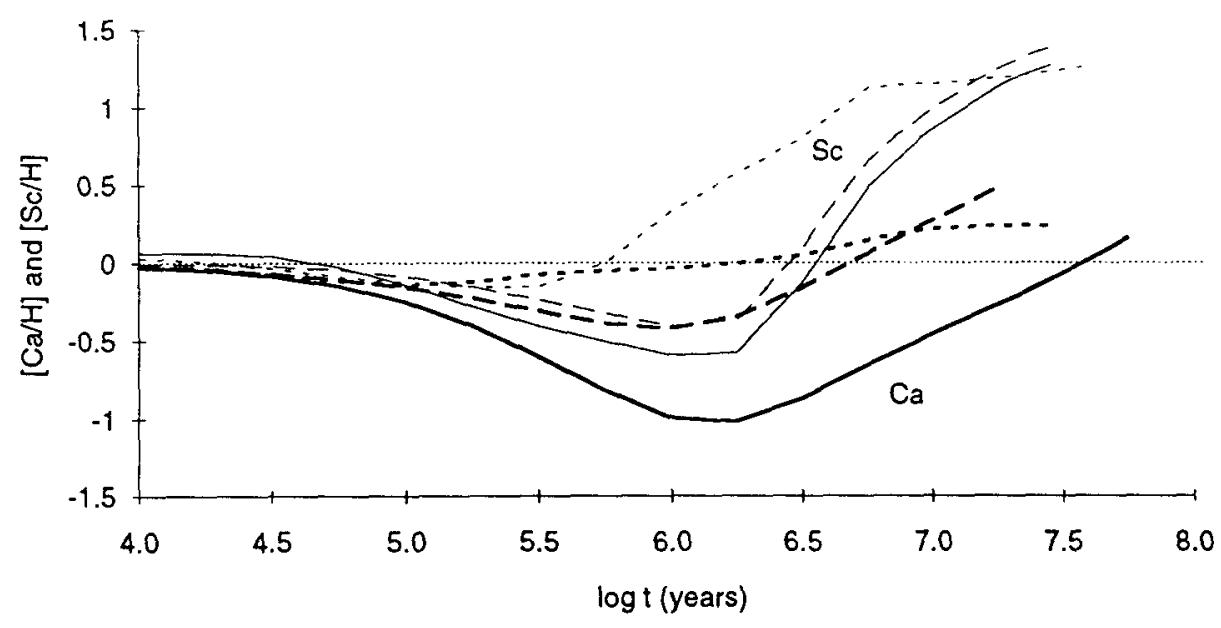

Fig. 2. Calcium and Scandium aboundances, $\mathrm{T}_{\text {eff }}=8000 \mathrm{~K}$. The thin lines are for $\mathrm{Sc}$ and the heavy ones are for $\mathrm{Ca}$. The continuous lines correspond to model 1, the dashed ones to model 2 and the long-dashed ones to model 3.

(iii) - The results we have obtained are not satisfactory in the case of aluminium which is always found to evolve toward underabundance in our computations. Observations show a rather slight systematic overabundance (Burkhart and Coupry, 1991). However, since radiative acceleration on $\mathrm{Al}$ almost balances gravity just below the convection zone, this element is very sensitive to errors on radiative accelerations. The methods we have employed for these computations are precise and efficient. But the important improvements on atomic data such as the one provided by the OPACITY project for instance, will have strong consequences on calculations of time-dependent diffusion

\section{ACKNOWLEDGEMENTS:}

We would like to thank A. Baglin for having kindly communicated the code computing the envelope models. We would like also to thank E. Schatzman who has estimated for us the possible extension of the bottom of the convection zone.

\section{REFERENCES}

ALECIAN, G.: 1986, Astron. Astrophys., 168, 204

ALECIAN, G., ARTRU, M.C.: 1990, Astron. Astrophys., 234, 323

ALECIAN, G.: 1990, IAU Symposium $\mathrm{N}^{\circ} 145$ on "Evolution of Stars: the photospheric abundance connection"

BURKHART, C., COUPRY, M.F.: 1991, Astron. Astrophys., 249, 205

KURUCZ, R.L., PEYTREMANN, E.: 1975 , A table of semi Empirical gf Values, Smithonian Astrophys. Obs. Spec. Rept. $\mathrm{N}^{\circ} 362$ 